\title{
EGU21-2805
}

https://doi.org/10.5194/egusphere-egu21-2805

EGU General Assembly 2021

(c) Author(s) 2022. This work is distributed under

the Creative Commons Attribution 4.0 License.

\section{Variations of VLF Wave Intensity Analyzed via Principal Component Analysis}

\author{
Barbora Bezděková ${ }^{1}$, František Němec ${ }^{1}$, Michel Parrot ${ }^{2}$, Jyrki Manninen ${ }^{3}$, Oksana Krupařová ${ }^{4}$, and \\ Vratislav Krupař $\check{r}^{5,6,4}$ \\ ${ }^{1}$ Faculty of Mathematics and Physics, Charles University, Prague, Czechia (baja@etranslator.biz) \\ ${ }^{2} \mathrm{LPC} 2 \mathrm{E} / \mathrm{CNRS}$, Orleans, France \\ ${ }^{3}$ Sodankyla Geophysical Observatory, Sodankyla, Finland \\ ${ }^{4}$ Department of Space Physics, Institute of Atmospheric Physics, Czech Academy of Sciences, Prague, Czech Republic \\ ${ }^{5}$ Universities Space Research Association, Columbia, MD, USA \\ ${ }^{6}$ Heliospheric Physics Laboratory, Heliophysics Division, NASA Goddard Space Flight Center, Greenbelt, MD, USA
}

Wave intensity measured in the very low frequency (VLF) range (up to $20 \mathrm{kHz}$ ) is typically represented using frequency-time spectrograms. Since the characterization of spectrogram main features and/or their direct comparison is a challenging task, we transform the measurements of the low-altitude DEMETER spacecraft using the principal component analysis (PCA). The present study is focused on both the physical interpretation of the first two principal components and their application to real physical problems. To understand the physical meaning of the first principal components, their scatter plot is constructed and discussed. Moreover, the dependence of the first principal component (PC1) coefficients on the geomagnetic activity and their seasonal/longitudinal variations are analyzed. The obtained distributions are well comparable with those obtained by previous studies for average wave intensities, indicating that the PC1 coefficients are directly related to the overall wave intensity. Furthermore, the variations of PC1 coefficients around interplanetary (IP) shock arrivals are analyzed, suggesting that the fast forward shock occurrence has the most significant effect. It is shown that the wave intensity variations depend on the wave intensity detected before the shock arrival. The shock strength and interplanetary magnetic field orientation are also important. To further demonstrate the adaptability of PCA, we use a similar method to analyze also ground-based VLF measurements performed by the Kannuslehto station located in northern Finland. 